Check for updates

Cite this: RSC Adv., 2017, 7, 42422

Received 7th July 2017

Accepted 28th August 2017

DOI: $10.1039 / c 7 r a 07479 d$

rsc.li/rsc-advances

\section{Decoration of chitosan microspheres with Brønsted heteropolyacids and Lewis ion Ti: trifunctional catalysts for esterification to biodiesel $\uparrow$}

\begin{abstract}
Tong Tong, Yiming Li, Rui Hou, Xiaohong Wang (D) * and Shengtian Wang
$\mathrm{H}_{3} \mathrm{PW}_{12} \mathrm{O}_{40}$ is a commonly used Brønsted acid catalyst in esterification and transesterification reactions to produce biodiesel, whose homogeneous form and single acid sites lead to difficulties in separation and relatively less activity. Herein, the water-insoluble and multifunctional active sites based on $\mathrm{H}_{3} \mathrm{PW}_{12} \mathrm{O}_{40}$, chitosan and $\mathrm{Ti}^{4+}$ had been fabricated giving $\mathrm{H}_{3} \mathrm{PW}_{12} \mathrm{O}_{40} / \mathrm{Ti} /$ chitosan tri-functional hybrids. Such hybrids exhibited higher activity in esterification reactions due to the existence of Brønsted acid from $\mathrm{H}_{3} \mathrm{PW}_{12} \mathrm{O}_{40}$, Lewis acid from $\mathrm{Ti}^{4+}$, and base sites from the $-\mathrm{NH}_{2}$ group of chitosan, and all also due to the generation of pores in chitosan through introduction of the Ti ions. Furthermore, $\mathrm{H}_{3} \mathrm{PW}_{12} \mathrm{O}_{40} / \mathrm{Ti} /$ chitosan acted as heterogeneous catalysts and could be separated for reuse at least six times without significant loss of activity and with little leaching of $\mathrm{Ti}^{4+}$ and $\mathrm{H}_{3} \mathrm{PW}_{12} \mathrm{O}_{40}$ from the support chitosan.
\end{abstract}

\section{Introduction}

Heteropolyacids (HPAs) are discrete early transition metal-oxide clusters with a variety of compositions and structures, which have been applied in many fields of science. ${ }^{1}$ HPAs are wellknown super-strong acids for both homogeneous and heterogeneous acid-catalyzed reactions. ${ }^{2}$ The free acidic forms of HPWs with Keggin structure, especially 12-tungstophosphoric acid $\left(\mathrm{H}_{3} \mathrm{PW}_{12} \mathrm{O}_{40}, \mathrm{HPW}\right)$, have attracted much attention for chemical conversions ${ }^{3}$ because of their strong Brønsted acidity and higher stability. During these years, fabrication of multifunctional HPAs have attracted much attention due to their wide applications in one-pot transformation or tandem reactions of organic substrates. ${ }^{4,5}$ Artificial HPA materials functionalized by acid-base synergy and cooperative effects are relatively less addressed and stimulate a real challenge. The first report on bifunctional HPAs catalysis with acidic and basic sites was reported by N. R. Shiju and co-workers, demonstrating that site-isolated amine and phosphotungstic acid groups were loaded on mesoporous silica, ${ }^{6}$ but the Brønsted acidity of HPAs and the base property of the host were suppressed by each other. Other bifunctional HPAs had been prepared using layered double hydroxides as the base source and HPAs as the acidic one. ${ }^{7}$ During our research work on biomass conversion catalyzed by HPAs, we designed and prepared acid-base HPAs using amino acids as base groups, ${ }^{4}$ which were used as

Key Lab of Polyoxometalate Science of Ministry of Education, Northeast Normal University, Changchun 130024, P. R. China. E-mail: wangxh665@nenu.edu.cn; Tel: +86-431-85099759

† Electronic supplementary information (ESI) available. See DOI: 10.1039/c7ra07479d bifunctional catalysts in transesterification and esterification reactions. Nevertheless, there is still plenty room for fabrication different bifunctional HPAs with spatially isolated multiple active sites and easy regeneration, which can promote simultaneous reactions.

Biodiesel is a viable alternative to replace petrodiesel, and it has become more attractive due to the environmental concerns. $^{8}$ The second-generation biodiesel is normally considered to be obtained from nonedible oils such as castor, Jatropha, microalgae, and animal fats (e.g. tallow and yellow grease). By now, an interesting alternative for low cost biodiesel production is the utilization of low quality raw materials as feedstocks, especial waste cooking oil (WCO) obtained from restaurants or from houses with higher contents of free fatty acids and water. ${ }^{9-11}$ Waste cooking oil, which is available cheaply, is an attractive starting material that can help in improving the economic feasibility of biodiesel especially for China or India. ${ }^{12}$ However, there are some drawbacks in conversion of such low-quality feedstocks - impossibility of converting free fatty acid (FFA) to FAME and soap formation catalyzed by base, and unavailability for mineral acid due to the existence of the high contents of water. ${ }^{13}$ In this concept, the heterogeneous acid catalysis process is expected to be an effective biodiesel production process with some benefits: insensitivity to FFA and water contents, simultaneous occurrence of esterification and transesterification, no further washing treatment for generated soup, easy separation of the catalyst from the reaction medium, low contamination of the product, recyclability of the catalyst, and no corrosion problem. ${ }^{14}$ However, their application usually suffer from inferior activity due to some disadvantages: declining acid strength, non-uniform dispersion of acid or base sites on the solid 
surface, mass transferring difficulty at the interface, or overlapped exposure of the acid sites. ${ }^{15}$ Therefore, an ideal solid acid catalyst for biodiesel production should have high stability, isolated acid sites, large pores, hydrophobic surface, multifunctional sites with coordination effect, recyclability and reusability. ${ }^{15,16}$ In addition, the existence of Lewis acid on the solid catalysts could provide synergistic effect on esterification reactions. ${ }^{17,18}$ From the literature, it is noted that catalysts with Lewis acid sites such as $\mathrm{Zn}, \mathrm{Ag}$, Sn could ${ }^{\mathbf{1 9}}$ gave the higher conversion of ester. In our previous work, $\mathrm{PW}_{11} \mathrm{TiO}_{40}{ }^{5-}$ anion had been prepared and showed promoting effect on esterification reaction..$^{20}$ Recently, some methods have been developed to design multifunctional solid catalysts to achieve esterification and transesterification occur simultaneously. ${ }^{15}$

Herein, we designed to synthesize bifunctional HPAs through loading Brønsted $\mathrm{HPW} \mathrm{H}_{3} \mathrm{PW}_{12} \mathrm{O}_{40}$ on chitosan to form acid-base hybrids HPW/chitosan. Chitosan has been proposed to be an ideal support material with weak basic properties due the existence of free amino groups. ${ }^{21} \mathrm{HPW}$ reacted with some of the amino groups, hence were loaded on the matrix of chitosan. And other amino groups without interacting with HPW were contribution to the basic property for HPW/chitosan. The acidity and basicity could be controlled through the component of HPW and chitosan. Furthermore, Lewis centres were introduced by introduction of Ti ion into the matrix of chitosan. Hence, trifunctional active sites based on heteropolyacid HPW/ $\mathrm{Ti} /$ chitosan were fabricated through the electrostatic interaction between amino group in chitosan and HPW molecules, coordination bond between $-\mathrm{NH}_{2}$ and $\mathrm{Ti}$ ions. The strong interaction between HPW, Ti and chitosan confirmed the less leaching of active sites from the support. Furthermore, this chitosan matrix did not dissolve in methanol, which could be easily separate from the reaction mixture for reuse. As-prepared HPW/ $\mathrm{Ti} /$ chitosan was evaluated in esterification of FFAs for production of biodiesel.

\section{Experimental}

\section{Materials}

$\mathrm{H}_{3} \mathrm{PW}_{12} \mathrm{O}_{40} \cdot 23 \mathrm{H}_{2} \mathrm{O}$ was prepared according to ref. 22. All chemicals of AR grade were attained from commercial suppliers without further purification.

\section{Preparation of catalysts}

In a 3-necked flask, urea $(0.1 \mathrm{~mol})$ and titanium sulfate $(0.0125$ mol) were dissolved in $100 \mathrm{~mL}$ of distilled water and stirred for $1 \mathrm{~h}$ at $333 \mathrm{~K}$. Acetic acid solution $(5 \%, 50 \mathrm{~mL})$ containing chitosan $(2 \%)$ was subsequently added to the flask. The $\mathrm{pH}$ was adjusted to 7 using ammonia, and then the mixture was stirring for about $3 \mathrm{~h}$. The powder was centrifuged to be separated and washed with water to separate unreacted urea and $\mathrm{Ti}\left(\mathrm{SO}_{4}\right)_{2}$. Finally, the powder was dried at $353 \mathrm{~K}$ for $6 \mathrm{~h}$ to give Ti functionalized chitosan (Ti/chitosan) ${ }^{23}$ with yield of $84 \%$.

$\mathrm{Ti} /$ chitosan $(0.48 \mathrm{~g})$ and $\mathrm{H}_{3} \mathrm{PW}_{12} \mathrm{O}_{40}(0.2 \mathrm{~g})$ were dissolved in dilute hydrochloric acid solution ( $1 \mathrm{M}, 20 \mathrm{~mL})$ together with vigorous stirring at room temperature for $6 \mathrm{~h}$. Then the powder was separated through centrifuge and dried at $303 \mathrm{~K}$ for $24 \mathrm{~h}$ to give HPW functionalized chitosan (HPW/Ti/chitosan) with yield of $78 \%$ as HPW (29 wt $\%) / \mathrm{Ti} /$ chitosan.

Other solid catalysts containing different amount of HPW was prepared using the same procedure except with various usage of HPW as $0.142 \mathrm{~g}, 0.166 \mathrm{~g}, 0.250 \mathrm{~g}$ and $0.334 \mathrm{~g}$, respectively.

\section{Determination of the acidic and basic properties}

Two titration methods were put into use to figure up the total acid content of the catalysts. Firstly, $0.05 \mathrm{~g}$ catalyst was put into $45 \mathrm{~mL}$ distilled water and stirring the mixture for $3 \mathrm{~h}$. The concentration of total acid was measured by titration with diluted $n$-butylamine in distilled water $(0.05 \mathrm{M})$ with the indicator anthraquinone $\left(\mathrm{p} K_{\mathrm{a}}=-8.2\right)$. And then IR spectra of pyridine adsorption worked for recognizing the Lewis and Brønsted acid sites. Pyridine was adsorbed by catalysts specimens for $12 \mathrm{~h}$ under the condition of vacuum and $60{ }^{\circ} \mathrm{C}$. The capacity of acidity was calculated by Lambert-Beer formula: ${ }^{24} \mathrm{~A}$ $=(\varepsilon \times W \times c) / S$. $\left(A\right.$ : absorbance, $\mathrm{cm}^{-1}, \varepsilon$ : extinction coefficient, $\mathrm{m}^{2} \mathrm{~mol}^{-1}, W$ : the sample weight, $\mathrm{kg}, c$ : the concentration of acid, $\mathrm{mmol} \mathrm{g}^{-1}, S$ : the sample disk area, $\mathrm{m}^{2}$ ). Base content of the catalysts was also obtained by titration and the pretreatment was the same as acid. Then the titration was proceeded with acetic acid in distilled water $(0.05 \mathrm{M})$ with the 2-(4-dimethylaminophenylazo) benzoic acid $\left(\mathrm{p} K_{\mathrm{a}}=4.76\right)$.

In addition, another titration method with automatic potentiometric titrator was done as $0.02 \mathrm{~g}$ catalyst was put into $40 \mathrm{~mL}$ acetonitrile solution with stirring intensely for $3 \mathrm{~h}$ and then the obtained suspension was titrated with diluted $n$ butylamine in acetonitrile $(0.1 \mathrm{M})$. The titration end-point could be received from potentiometric titration curve so that the total acid content of the catalysts could be calculated. In a similar way, the base content was measured. Putting $0.02 \mathrm{~g}$ catalyst into ethanol solution and stirring for $3 \mathrm{~h}$. Then the mixture was titrated with benzoic acid in ethanol $(0.02 \mathrm{M})$.

\section{Esterification reaction}

Esterification reaction was carried out in a $50 \mathrm{~mL}$ three-necked glass flask charged with cooled condenser and a mechanical stirrer at $65{ }^{\circ} \mathrm{C}$. In a typical experiment, the reactor was loaded with $2.5 \mathrm{~g}$ of palmitic acid, a certain amount of anhydrous methanol, and a certain amount of catalyst. Samples were taken periodically, and tested to determine the conversion of palmitc acid in gas chromatography (GC). After finishing reaction, the catalyst was separated by centrifugation, washed with methanol to remove the remaining reactants and water, then dried in air and reused for the next experiment. And the leaching of the solid catalyst into the mixture was measured the $\mathrm{W}$ contents by ICP.

\section{Instrument}

The acidic and basic properties were measured on a ZDJ-4B automatic potentiometric titrator. FTIR spectra (4000$500 \mathrm{~cm}^{-1}$ ) were recorded on a Nicolet Magna 560 IR spectrometer. A Leeman corporation inductively coupled plasma 
(ICP) emission spectrometer and a PE 2400 CHN elemental analyzer was used to determine the contents of elements in catalysts. XPS was performed by an Escalab-MK II photoelectronic spectrometer with $\mathrm{Al} \mathrm{K} \alpha(1200 \mathrm{eV}) .{ }^{31} \mathrm{P}$ MAS NMR spectra of the catalysts were obtained using a Bruker AM500 spectrometer at 202.5 MHz. Nitrogen adsorption-desorption isotherms and Brunauer-Emmet-Teller (BET) surface area were measured on an V-Sorb2800P surface analyzer. X-ray diffraction (XRD) patterns of the catalysts were obtained on Rigaku Dmax $2000 \mathrm{X}$-ray diffractometer with $\mathrm{Cu} \mathrm{K} \alpha$ radiation $(\lambda$ $=0.154178 \mathrm{~nm}$ ) (Rigaku Corporation). The energy dispersive Xray spectrometry (EDS) elemental mapping was given on a HITACHI SU8010 Field-emission scanning electron microscopy. The concentrations of esters were determined periodically on Agilent Technologies 7820A GC system fitted with capillary column $(30 \mathrm{~m} \times 0.25 \mathrm{~mm})$ and flame ionization detector.

\section{Results and discussion}

\section{Preparation of trifunctional HPW/Ti/chitosan}

Chitosan is a kind of polysaccharide macromolecules as hierarchical supports for catalysts, which was used to fabricate acid-base bifunctional catalysts. ${ }^{25-27} \mathrm{M}$. Yamada had used the amino groups in chitosan to load heteropolyacids $\mathrm{H}_{3} \mathrm{PW}_{12} \mathrm{O}_{40}$ through the interaction of $-\mathrm{NH}_{3}{ }^{+}$and $\mathrm{PW}_{12} \mathrm{O}_{40}{ }^{3-}$, which exhibited catalytic activity in epoxidation of allylic alcohols. ${ }^{28}$ And strong electrostatic interaction between HPW anion and amino group in chitosan confirmed no leaching of HPW from support. Using the same synthetic strategy, here we fabricated trifunctional HPW/Ti/chitosan containing Brønsted acid, Lewis acid and base sites. Firstly, Ti metal ions were loaded on chitosan matrix through coordinate interaction following the equation:

$$
\text { Chitosan- } \mathrm{NH}_{2}+\mathrm{Ti}\left(\mathrm{SO}_{4}\right)_{2} \rightarrow \text { chitosan- } \mathrm{NH}_{2} \cdots \mathrm{Ti}
$$

Secondly, HPW molecules were anchored on chitosan matrix by mixing the Ti/chitosan and HPW solutions through electrostatic interaction between $\mathrm{PW}_{12} \mathrm{O}_{40}{ }^{3-}$ and $\mathrm{NH}_{3}{ }^{+}$as HPW $\cdots$ $\mathrm{NH}_{3}{ }^{+}$-chitosan- $\mathrm{NH}_{2} \cdots \mathrm{Ti}$ (Scheme 1).

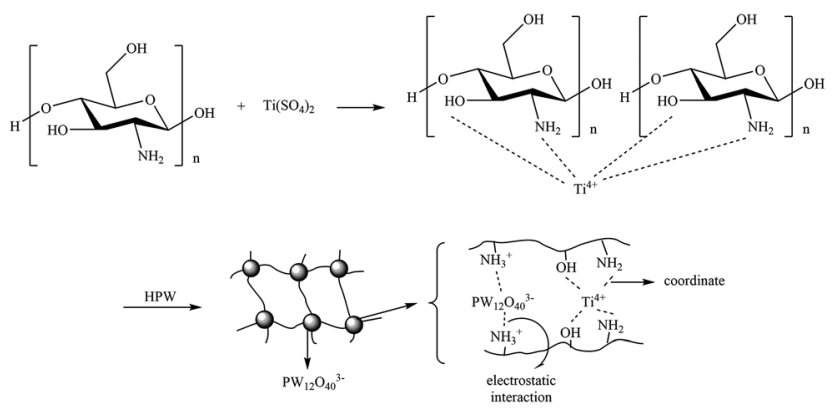

Scheme 1 The preparation of HPW/Ti/chitosan hybrids.

\section{Characterization of catalysts}

From the result of elemental analysis, the loading amounts of $\mathrm{H}_{3} \mathrm{PW}_{12} \mathrm{O}_{40}$ on chitosan were $23 \mathrm{wt} \%, 26 \mathrm{wt} \%, 29 \mathrm{wt} \%, 34 \mathrm{wt} \%$ and $41 \mathrm{wt} \%$, respectively (Table 1). And the amount of Ti was about $19 \mathrm{wt} \%, 18 \mathrm{wt} \%, 17 \mathrm{wt} \%, 16 \mathrm{wt} \%$ and $15 \mathrm{wt} \%$, respectively.

The IR spectra of HPW/Ti/chitosan were investigated (Fig. 1). For Ti/chitosan, the band at $1620 \mathrm{~cm}^{-1}$ and $590 \mathrm{~cm}^{-1}$ were assigned to vibration of $\mathrm{Ti}-\mathrm{NH}_{2}$ bond, ${ }^{29}$ and $\mathrm{Ti}-\mathrm{O}$ bond, ${ }^{30}$ respectively, determining that $\mathrm{Ti}$ ions were loaded on the matrix of chitosan through coordinate interaction between $\mathrm{Ti}$ and $-\mathrm{OH}$ groups and $\mathrm{Ti}$ and $-\mathrm{NH}_{2}$ as well in chitosan. In addition, the vibration of $\mathrm{O}-\mathrm{H}$ in chitosan was revealed at $1020 \mathrm{~cm}^{-1}$ and in $\mathrm{Ti} /$ chitosan was presented at $1068 \mathrm{~cm}^{-1}$. And some of $-\mathrm{NH}_{2}$ vibration peak also shifted from $1545 \mathrm{~cm}^{-1}$ to $1522 \mathrm{~cm}^{-1}$ belongs to $-\mathrm{NH}_{3}{ }^{+}$. Such shifts also confirmed the existence of coordinate interaction between $\mathrm{Ti}^{4+}$ and $-\mathrm{NH}_{2}$ and $-\mathrm{OH}$ in chitosan. For $\mathrm{H}_{3} \mathrm{PW}_{12} \mathrm{O}_{40}$, the IR stretching vibrations found in the range of 700-1100 $\mathrm{cm}^{-1}$ were assigned to the Keggin unit of $\mathrm{PW}_{12} \mathrm{O}_{40}{ }^{3-}$, which correspond to vibration of $\mathrm{P}-\mathrm{O}_{\mathrm{a}}\left(1073 \mathrm{~cm}^{-1}\right)$, $\mathrm{W}=\mathrm{O}_{\mathrm{d}}\left(957 \mathrm{~cm}^{-1}\right), \mathrm{W}-\mathrm{O}_{\mathrm{C}}-\mathrm{W}$ and $\mathrm{W}-\mathrm{O}_{\mathrm{b}}-\mathrm{W}\left(877\right.$ and $\left.745 \mathrm{~cm}^{-1}\right)$, respectively. After being loaded on chitosan, these four peaks were also found with some shifts of the peak positions. The peaks for $\mathrm{W}=\mathrm{O}_{\mathrm{d}}, \mathrm{W}-\mathrm{O}_{\mathrm{C}}-\mathrm{W}$ and $\mathrm{W}-\mathrm{O}_{\mathrm{b}}-\mathrm{W}$ shifted to higher wavenumbers, indicating the existence of electrostatic interaction between HPW anion and chitosan. ${ }^{31}$ Same results confirmed the structural integrity of HPW and stability of HPW and $\mathrm{Ti}$ in chitosan.

The structural integrity of the Keggin unit in the HPW (29 wt\%)/Ti/chitosan hybrids was further confirmed by ${ }^{31} \mathrm{P}$ MAS NMR (Fig. 2a). One peak with a shoulder for HPW/Ti/chitosan was observed at $-16.92 \mathrm{ppm}$, which was attributed to the resonance of $\mathrm{PO}_{4}$ unit within the bulk $\mathrm{H}_{3} \mathrm{PW}_{12} \mathrm{O}_{40}$ similar to its parent $^{32}(-15.6 \mathrm{ppm})$. The shoulder and some shift were assigned to interaction between chitosan and $\mathrm{H}_{3} \mathrm{PW}_{12} \mathrm{O}_{40}$, which confirmed less leaching of $\mathrm{H}_{3} \mathrm{PW}_{12} \mathrm{O}_{40}$ from the support chitosan during the catalytic reactions.

The XPS analysis mainly reflects the composition and chemical elementary state of the surface and the inferior surface of sample. ${ }^{33}$ XPS survey spectrum and the corresponding high-resolution of the catalysts were shown in Fig. 3a. Compared to chitosan, new absorption peaks at $458.6 \mathrm{eV}$ and $464.3 \mathrm{eV}$ correspond to $\mathrm{Ti} 2 \mathrm{p}_{3 / 2}$ and $\mathrm{Ti} 2 \mathrm{p}_{1 / 2}$ in $\mathrm{Ti} /$ chitosan $^{34}$ were found due to the introduction of $\mathrm{Ti}$ into the chitosan matrix (Fig. 3c), which existed as $\mathrm{Ti}^{4+}$. And the $\mathrm{N} 1 \mathrm{~s}$ gave two peaks at $399.70 \mathrm{eV}$ and $401.51 \mathrm{eV}$, respectively (Fig. 3b), which the peak at around $399.70 \mathrm{eV}$ was originated from $\mathrm{NH}_{2}$ from chitosan. And another peak at $401.51 \mathrm{eV}$ was due to the coordinated $\mathrm{N}$ to $\mathrm{Ti}$ $\left(\mathrm{NH}_{2} \rightarrow \mathrm{Ti}\right) .^{35}$ Therefore, there were two species of nitrogen species existence, i.e. coordinated $\mathrm{N}$ and unreacted $\mathrm{N}$. This confirmed that $\mathrm{Ti}$ cation was successfully introduced into chitosan through coordination interaction. For HPW/Ti/chitosan (Fig. 3d), the $\mathrm{N}$ 1s also gave two peaks at $400.33 \mathrm{eV}$ and $401.86 \mathrm{eV}$, respectively, which were attributed to $\mathrm{NH}_{2}$ from chitosan and $-\mathrm{NH}_{2}$ to Ti or $\mathrm{NH}_{3}{ }^{+}$, respectively. This indicated the formation of $\mathrm{HPW} / \mathrm{Ti} /$ chitosan through electrostatic 


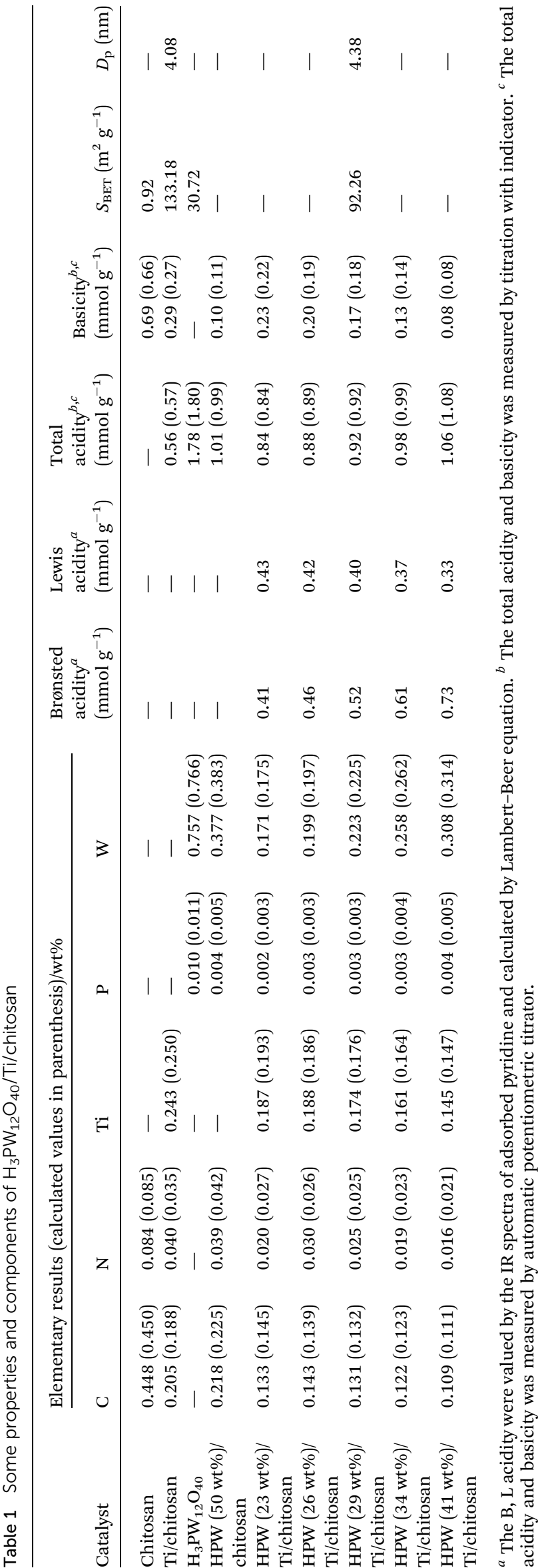

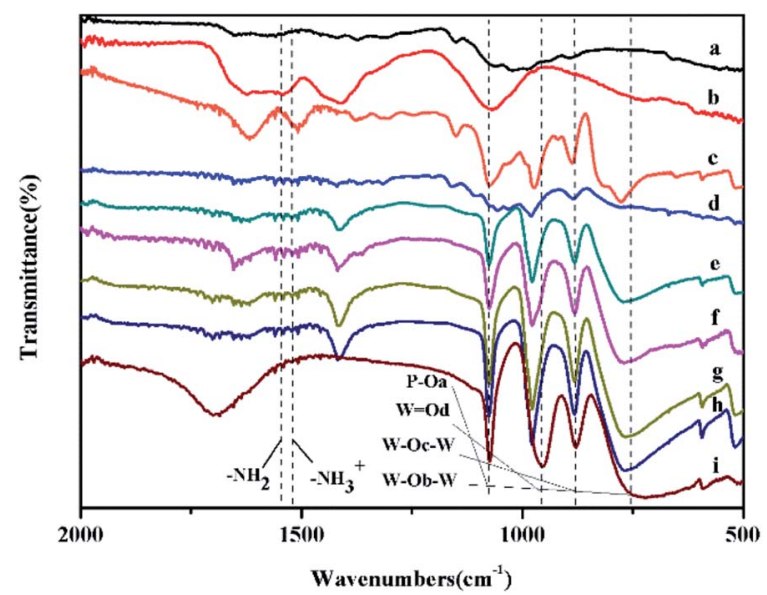

Fig. 1 IR spectra of (a) chitosan, (b) Ti/chitosan, (c) HPW (50 wt\%)/ chitosan, (d) HPW (23 wt\%)/Ti/chitosan, (e) HPW (26 wt\%)/Ti/chitosan, (f) HPW (29 wt\%)/Ti/chitosan, (g) HPW (34 wt\%)/Ti/chitosan, (h) HPW (41 wt\%)/Ti/chitosan, and (i) $\mathrm{H}_{3} \mathrm{PW}_{12} \mathrm{O}_{40}$.

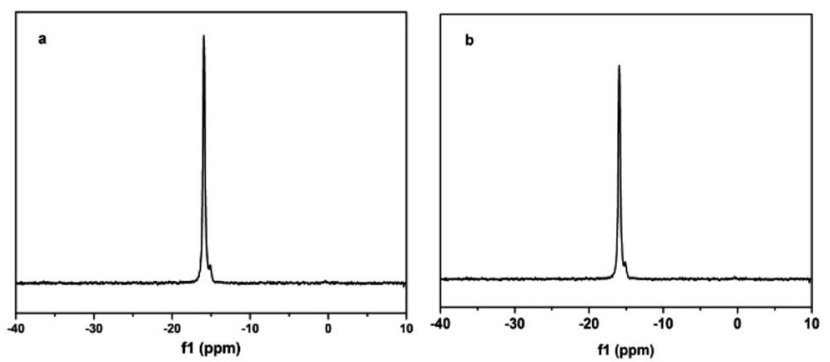

Fig. $2{ }^{31} \mathrm{P}$ MAS NMR spectra of HPW (29 wt\%)/Ti/chitosan before (a) and after catalytic reaction (b).

interactions. Meanwhile, two new peaks at $35.38 \mathrm{eV}$ and $37.45 \mathrm{eV}$ were found, which the new peaks were attributed to the $\mathrm{W}^{6+}$ from HPW. ${ }^{36}$ As result, HPW/Ti/chitosan triple solid had been prepared as HPW $\cdots \mathrm{NH}_{3}{ }^{+}$-chitosan- $\mathrm{NH}_{2} \cdots$ Ti.

Nitrogen sorption analysis for chitosan, Ti/chitosan, HPW and HPW (29 wt\%)/Ti/chitosan were done to analyze the surface area and pore characters (Table 1 and Fig. S1 $\dagger$ ). It can be seen that native chitosan and HPW exhibited lower BET surface area and no porous characters. ${ }^{37}$ However, the surface areas were found to be 133.18 and $92.26 \mathrm{~m}^{2} \mathrm{~g}^{-1}$ corresponding to $\mathrm{Ti} /$ chitosan and HPW (29 wt\%)/Ti/chitosan, respectively. And pore diameters were $4.08 \mathrm{~nm}$ and $4.38 \mathrm{~nm}$, respectively. These new generations for surface area and porous characters were supposed to the introduction of $\mathrm{Ti}^{4+}$ to chitosan matrix.

The acidic and basic properties for chitosan, Ti/chitosan and a series of HPW/Ti/chitosan were measured using different methods (Table 1). And these two methods did not give significant difference for total acidity and basicity. It can be seen that native chitosan only exhibited basic property with basic strength as $0.69 \mathrm{mmol} \mathrm{g}^{-1}$. For Ti/chitosan, the basicity was decreased to $0.29 \mathrm{mmol} \mathrm{g}^{-1}$ and Lewis acidic strength was enhanced to $0.56 \mathrm{mmol} \mathrm{g}^{-1}$ due to the introduction of Lewis acid metal Ti. 

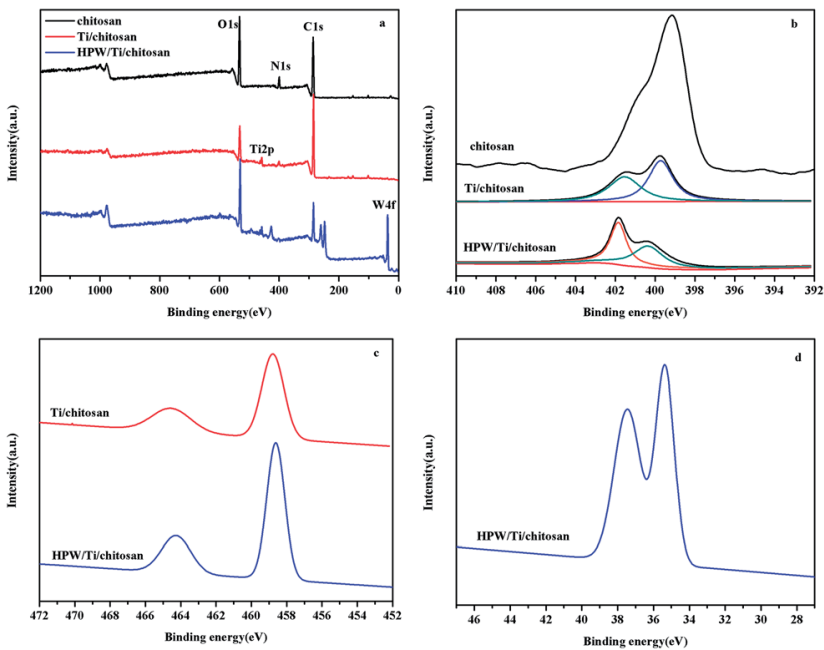

Fig. 3 XPS surveys and the corresponding high-resolution (a), N 1s (b), $\mathrm{Ti} 2 \mathrm{p}(\mathrm{c})$, and $\mathrm{W} 4 \mathrm{f}(\mathrm{d})$ core level spectra of the chitosan, Ti/chitosan and HPW (29 wt\%)/Ti/chitosan.

HPW/Ti/chitosan exhibited both acidic and basic properties, as well as double Brønsted acidity and Lewis acidity. The total acidity depended on the different weight ratios between HPW : Ti : chitosan as HPW (41 wt\%)/Ti/chitosan $\left(1.06 \mathrm{mmol} \mathrm{g}^{-1}\right)>$ HPW $(34 \mathrm{wt} \%) / \mathrm{Ti} /$ chitosan $\left(0.98 \mathrm{mmol} \mathrm{g}^{-1}\right)$ $>$ HPW (29 wt\%)/Ti/chitosan (0.92 $\left.\mathrm{mmol} \mathrm{g}^{-1}\right)>$ HPW (26 wt\%)/ $\mathrm{Ti} /$ chitosan $\left(0.88 \mathrm{mmol} \mathrm{g}^{-1}\right)>$ HPW (23 wt\%)/Ti/chitosan $\left(0.84 \mathrm{mmol} \mathrm{g}^{-1}\right)$. It can be concluded that higher amount of HPW in hybrids, higher acid capacity would be owned for HPW/ $\mathrm{Ti} /$ chitosan materials. Meanwhile, the basic capacity presented the opposite trend to acidic strength. All HPW/Ti/chitosan exhibited Lewis acidic sites due to the existence of $\mathrm{Ti}^{4+}$, which were determined by IR spectra of pyridine adsorption (Fig. 4). It can be seen that characteristic peaks at around $1540 \mathrm{~cm}^{-1}$ and $1450 \mathrm{~cm}^{-1}$ were attributed to the materials with Brønsted and Lewis acidic sites. ${ }^{24}$ Introduction of $\mathrm{Ti}^{4+}$ and HPW to chitosan

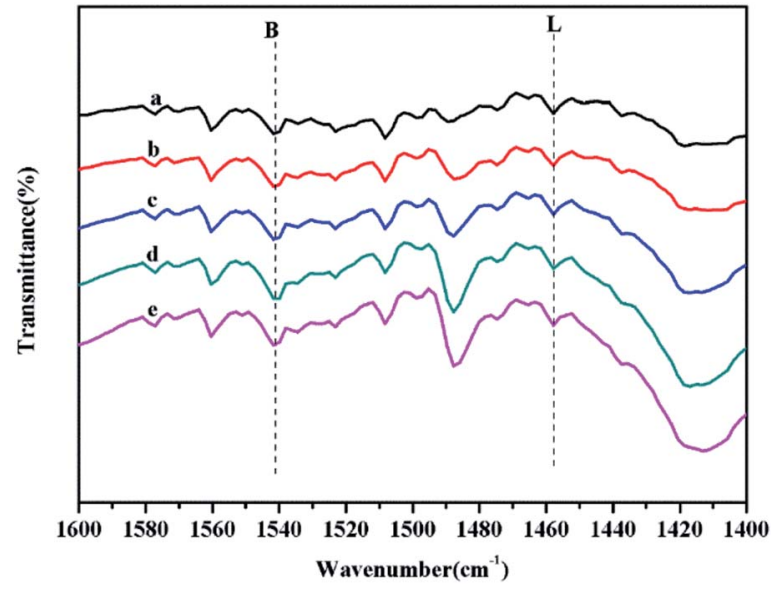

Fig. 4 IR spectra of pyridine adsorption of (a) HPW (23 wt\%)/Ti/chitosan, (b) HPW (26 wt\%)/Ti/chitosan, (c) HPW (29 wt\%)/Ti/chitosan, (d) HPW (34 wt\%)/Ti/chitosan, and (e) HPW (41 wt\%)/Ti/chitosan. resulted in tri-functional acid-base catalysts, and the acidic and basic distribution could be controlled by varying the different loading amount of HPW on Ti/chitosan.

\section{Catalytic activity}

Stimulated by the presence of both basic primary amino groups $\left(\mathrm{NH}_{2}\right)$ from chitosan, Brønsted acidity from HPW, and Lewis acidity by $\mathrm{Ti}$, we anticipated that this coexistence might lead to synergic and cooperative effect in catalysis. We selected an organic transformation (esterification), which is considered as sensible to the acid/base synergy.

In order to determine the different influence of active sites on esterification reaction of palmitic acid with methanol, the comparison experiments (Fig. 5) had been done using a various of catalysts including chitosan, Ti/chitosan, $\mathrm{H}_{3} \mathrm{PW}_{12} \mathrm{O}_{40}, \mathrm{HPW}$ (50 wt\%)/chitosan, HPW (23 wt\%)/Ti/chitosan, HPW (26 wt\%)/ Ti/chitosan, HPW (29 wt\%)/Ti/chitosan, HPW (34 wt\%)/Ti/ chitosan, HPW (41 wt\%)/Ti/chitosan under the reaction conditions as $65{ }^{\circ} \mathrm{C}$, molar ratio of methanol/oil $=13: 1,5 \mathrm{wt} \%$ catalyst, $7 \mathrm{~h}$. It can be seen that without any catalysts, palmitic acid was esterified with methanol only giving $8.7 \%$ conversion. Using chitosan and $\mathrm{Ti} /$ chitosan the conversion of palmitic acid increased to $33.9 \%$ and $63.2 \%$, respectively. The TOF values $(\mathrm{TOF}=($ conversion $\% \times$ amount of palmitic acid $) /[$ actual active sites (acidity + basicity) $\times h] \mathrm{g} \mathrm{mmol}^{-1} \mathrm{~h}^{-1}$ ) based on chitosan and Ti/chitosan were 1.40 and $2.12 \mathrm{~g} \mathrm{mmol}^{-1} \mathrm{~h}^{-1}$, respectively. It can be concluded that for chitosan only showed lower activity in esterification, while Ti/chitosan with Lewis acid sites resulted in the increasing of TOF. This indicated that the Lewis acid metals played a positive effect on esterification reactions, while the base sites showed a little activity in esterification. Compared to chitosan, HPW/Chitosan presented increasing TOF as $2.20 \mathrm{~g} \mathrm{mmol}^{-1} \mathrm{~h}^{-1}$ under the same reaction conditions, showing that the introduction of Brønsted acid played a positive effect on esterification. And the esterification efficiency was

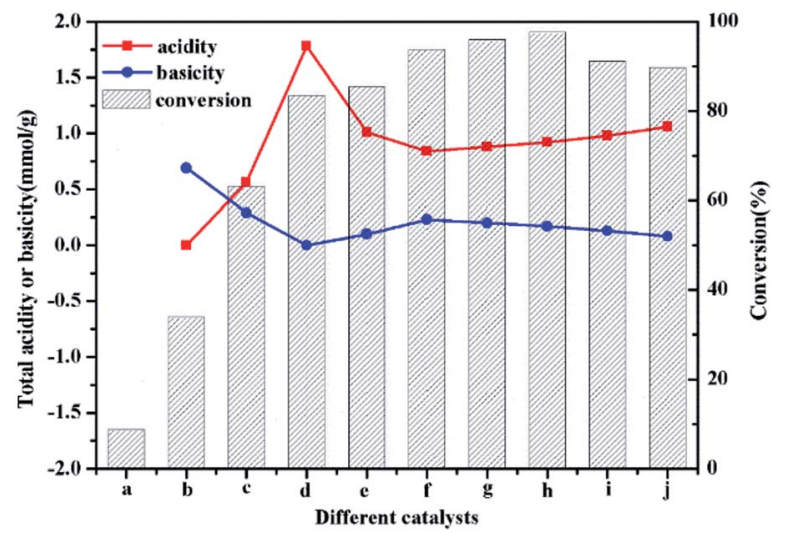

Fig. 5 The influence of total acidity or basicity on conversion by different catalysts. (a) Empty, (b) chitosan, (c) Ti/chitosan, (d) $\mathrm{H}_{3} \mathrm{PW}_{12} \mathrm{O}_{40}$, (e) HPW (50 wt\%)/chitosan, (f) HPW (23 wt\%)/Ti/chitosan, (g) HPW (26 wt\%)/Ti/chitosan, (h) HPW (29 wt\%)/Ti/chitosan, (i) HPW (34 wt\%)/Ti/chitosan, and (j) HPW (41 wt\%)/Ti/chitosan. Reaction conditions: $65^{\circ} \mathrm{C}$, molar ratio of methanol/oil $=13: 1,5 \mathrm{wt} \%$ catalyst, $7 \mathrm{~h}$. 
increased significantly as adding $\mathrm{HPW} / \mathrm{Ti} /$ chitosan catalysts with ranging as $\mathrm{H}_{3} \mathrm{PW}_{12} \mathrm{O}_{40}<\mathrm{HPW}(41 \mathrm{wt} \%) / \mathrm{Ti} /$ chitosan $<\mathrm{HPW}$ (34 wt\%)/Ti/chitosan < HPW $(23 \mathrm{wt} \%) / \mathrm{Ti} /$ chitosan < HPW (26 wt\%)/Ti/chitosan < HPW (29 wt\%)/Ti/chitosan.

It can be seen that all $\mathrm{HPW} / \mathrm{Ti} /$ chitosan materials present higher activity than their parent $\mathrm{H}_{3} \mathrm{PW}_{12} \mathrm{O}_{40}$. HPW as strong Brønsted acid presented $83.5 \%$ conversion with TOF of $1.34 \mathrm{~g}$ $\mathrm{mmol}^{-1} \mathrm{~h}^{-1}$, which did not give higher conversion of palmitic acid than HPW/chitosan (TOF $=2.20 \mathrm{~g} \mathrm{mmol}^{-1} \mathrm{~h}^{-1}$ ) with smaller acidic capacity but with basicity. Such increase was contributed to the synergistic effect of multifunctional sites including Brønsted acid and base belonging to HPW/chitosan. Compared with HPW/chitosan, Ti/chitosan only exhibited Lewis acidity and almost the same basicity, which gave $63.2 \%$ conversion and $2.12 \mathrm{~g} \mathrm{mmol}^{-1} \mathrm{~h}^{-1}$. This result showed that the existence of Brønsted acid sites was rather essential for esterification between acid and methanol than that of Lewis acid sites. After combination of triple sites of Brønsted acid, Lewis acid and base, the acid conversion and TOF was enhanced significantly as $97.6 \%$ conversion with TOF as $2.56 \mathrm{~g} \mathrm{mmol}^{-1} \mathrm{~h}^{-1}$ being catalyzed by HPW (29 $\left.\mathrm{wt} \%\right) / \mathrm{Ti} /$ chitosan. Therefore, it can be concluded that synergistic effect of basicity on esterification would help more palmitic acid convert to ester. Furthermore, the catalytic activity for HPW/Ti/ chitosan materials depended on the loading amount of HPW on chitosan. The catalytic activity increased as the loading amount decreased from $23 \mathrm{wt} \%$ to $29 \mathrm{wt} \%$, which was attributed to increasing of HPW on chitosan resulted in increasing of Brønsted acid strength, hence the conversion of acid. Further increasing of HPW amount from $34 \mathrm{wt} \%$ to $41 \mathrm{wt} \%$, the efficiency decreased due to the large aggregating existence of HPW molecules on chitosan.

As a significant influencing factor, different substrate has crucial effect on the efficiency of the esterification reaction. For this purpose, a series of acids and alcohols was used as substrate and the efficiency for the conversion was shown in Fig. 6. A phenomenon could be observed that palmitic acid and methanol had the greatest conversion efficiency, while cinnamic acid and $n$-amyl alcohol performed with worst efficiency. That might be due to the steric-hindrance effect of the molecules. Superabundant branched chain and unsaturated bond obstructed free rotation of single bond, therefore, it impeded the contact of reactant so that displayed low conversion.

Generally, in order to obtain a higher conversion of esters, excess methanol is necessary because of the reverse reaction for esterification. From Fig. 7, it can be seen that changing the methanol/acid from $11: 1$ to $16: 1$ resulted in a significant effect of the ester yield. Obviously, at the methanol/acid ratio of $13: 1$, the conversion of the reaction reached the maximum of $97.6 \%$. By increasing the molar ratio continually, no improvement was observed. ${ }^{38}$ The best selection of the result was methanol/acid ratio of $13: 1$ being catalyzed by HPW (29 wt\%)/ $\mathrm{Ti} /$ chitosan.

During the esterification process, the mass of catalyst represented a critical parameter to attain a high yield. Experiments were carried out by changing the catalyst amounts from $3 \mathrm{wt} \%$ to $7 \mathrm{wt} \%$ and keeping a methanol/oil ratio of $13: 1$ for $7 \mathrm{~h}$ at
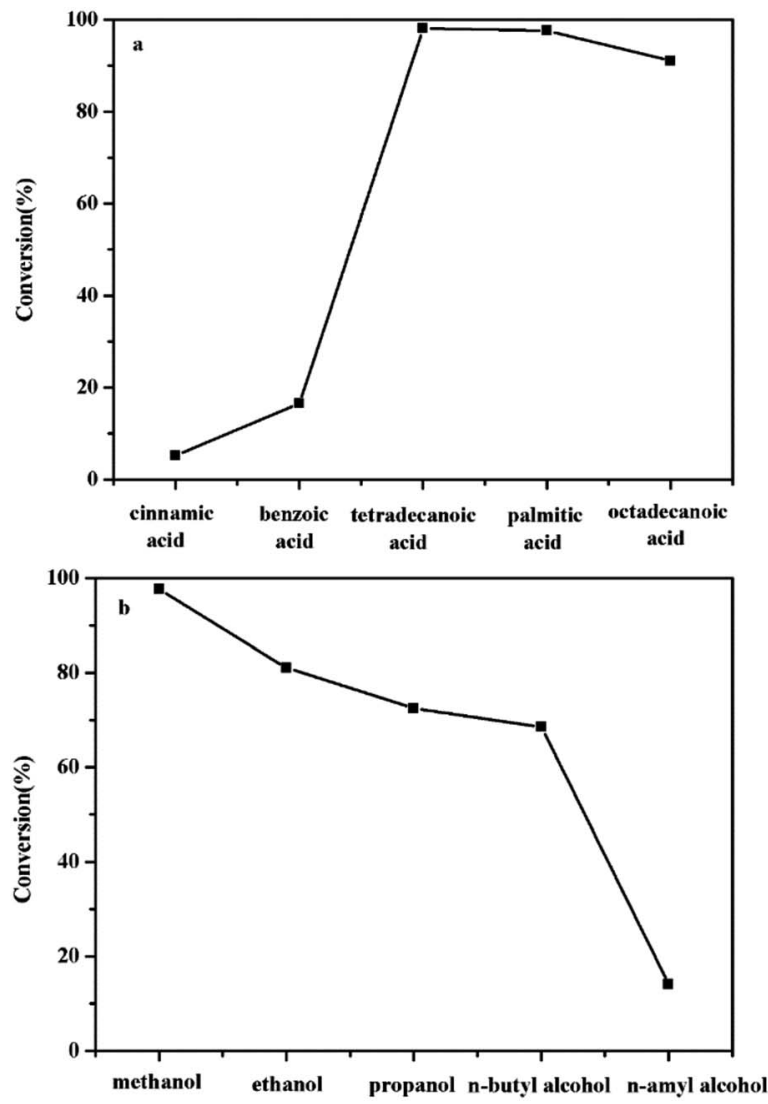

Fig. 6 The effect of different (a) acid and (b) alcohol in esterification reaction. Reaction conditions: $65^{\circ} \mathrm{C}$, molar ratio of alcohol/palmitic acid $=13: 1,5 \mathrm{wt} \%$ catalyst, $7 \mathrm{~h}$.

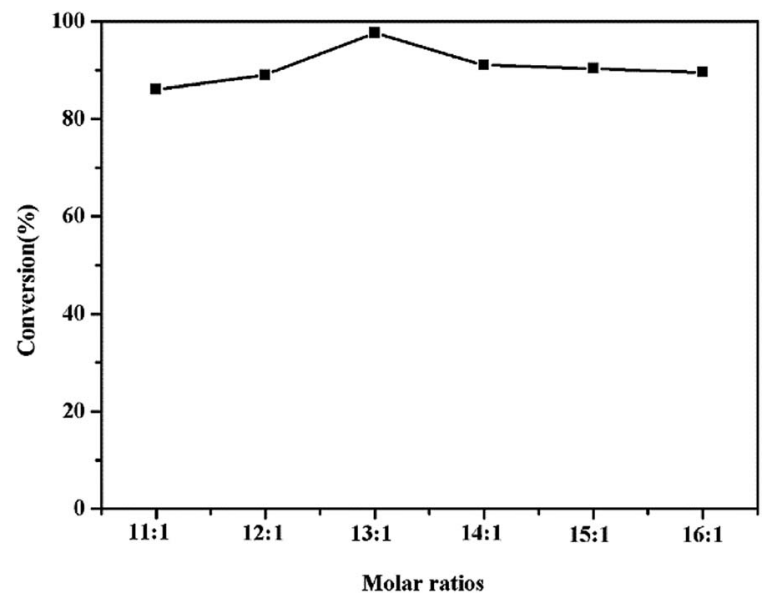

Fig. 7 The effect of methanol/palmitic acid molar ratio. Reaction conditions: $65^{\circ} \mathrm{C}, 5 \mathrm{wt} \%$ catalyst, $7 \mathrm{~h}$.

$65{ }^{\circ} \mathrm{C}$. Fig. 8 showed the effect of the catalyst amount to the conversion. It can be noticed that the conversion increased from $76.5 \%$ to $97.6 \%$ through increasing the catalyst from $3 \mathrm{wt} \%$ to $5 \mathrm{wt} \%$, then the conversion remained the same by further increasing the amount of the catalyst. ${ }^{39}$ When $5 \mathrm{wt} \%$ amount of catalyst was used, the acid-based catalysis process reached a maximum conversion of $97.6 \%$. 


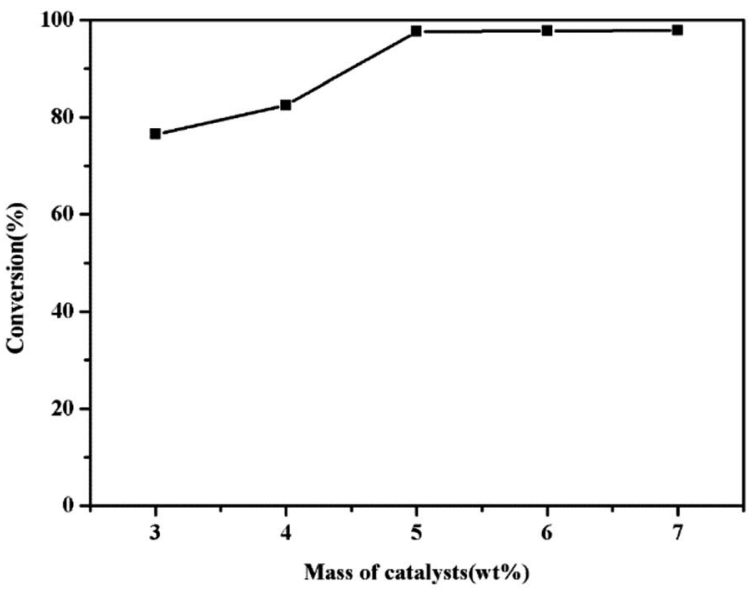

Fig. 8 The effect of mass of catalysts (wt\%). Reaction conditions: $65^{\circ} \mathrm{C}$, molar ratio of methanol/oil $=13: 1,7 \mathrm{~h}$.

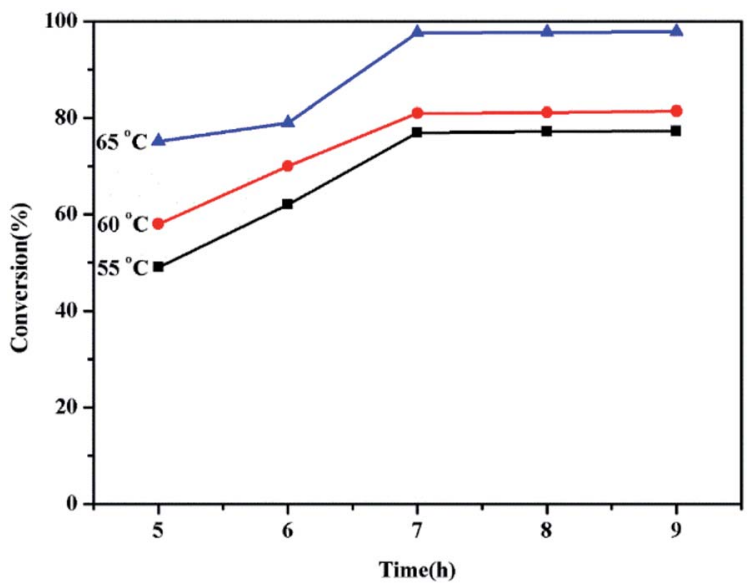

Fig. 9 The effect of reaction temperature and time. Reaction conditions: $5 \mathrm{wt} \%$ catalyst, molar ratio of methanol/oil $=13: 1$.

To explore the effect of temperature, the reaction was carried out at $55^{\circ} \mathrm{C}, 60{ }^{\circ} \mathrm{C}$ and $65^{\circ} \mathrm{C} . .^{40,41}$ The conversion of palmitic acid at different reaction temperatures was shown in Fig. 9. It can be seen that the conversion of palmitic acid increased with temperature from $55{ }^{\circ} \mathrm{C}$ to $65{ }^{\circ} \mathrm{C}$, which reached the maximum value at $65^{\circ} \mathrm{C}$. Under catalyzing, the esterification of acid to fatty acid ester can get the maximum conversion $97.6 \%$ at $65{ }^{\circ} \mathrm{C}$.

In order to determine the effect of reaction time, esterification reactions were carried out ranging from $5 \mathrm{~h}$ to $9 \mathrm{~h}$. It was found (Fig. 9) that the conversion of fatty acid increased before $7 \mathrm{~h}$ and reached the maximum $97.6 \%$ at $7 \mathrm{~h}$. Therefore, the optimum reaction time was reacted at $7 \mathrm{~h}$.

\section{Reusability of the catalyst}

From the viewpoint of practical applications of the catalyst, an important advantage of the solid catalyst is its recyclable usage and catalyst stability. The HPW/Ti/chitosan catalyst was easily separated from the mixture by centrifugation, and was washed with methanol for a few times to remove the polar compounds

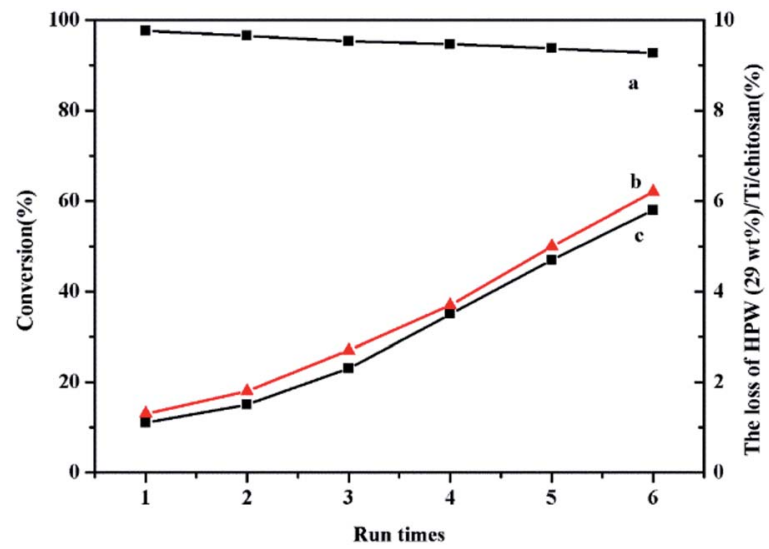

Fig. 10 In esterification the run times of catalyst. (a) Conversion, (b) the loss of $\mathrm{Ti}^{4+}$ in HPW $(29 \mathrm{wt} \%) / \mathrm{Ti} /$ chitosan, and (c) the loss of HPW in HPW (29 wt\%)/Ti/chitosan. Reaction condition: $65^{\circ} \mathrm{C}, 5 \mathrm{wt} \%$ catalyst, molar ratio of methanol/oil $=13: 1,7 \mathrm{~h}$.

then being dried in the air for reuse. After six reactions cycles, there was slightly change in the catalytic activity of the catalyst (Fig. 10). The leaching ratio of $\mathrm{Ti}^{4+}$ and HPW was only $6.2 \%$ and $5.8 \%$ after six cycles. ${ }^{42}$ The HPW kept the Keggin structure which can be proved by ${ }^{31} \mathrm{P}$ MAS NMR (Fig. 2). Furthermore, the IR spectra (Fig. S2 $\dagger$ ) with obvious feature peaks of $\mathrm{Ti}^{4+}$ and HPW after esterification reaction also demonstrated the stability of catalyst. The X-ray diffraction (XRD) and the Energy Dispersive $\mathrm{X}$-ray spectrometry (EDS) elemental mappings of HPW were also used to check the stability and components. From XRD (Fig. S3†), it can be seen that fresh HPW/Ti/Chitosan gave peaks at $9.63^{\circ}(110), 25.42^{\circ}(222), 34.42^{\circ}$ (332) and $37.73^{\circ}$ (510) with little shift corresponding to their parent HPW (JCPDS no. 752125), while intense peak at $25.76^{\circ}$ (101) was due to a characteristic peak of O-Ti-O (JCPDS no. 75-1537) and peaks of chitosan at $10.43^{\circ}$ and $21.00^{\circ}$ was also observed. After reaction, these peaks were found, indicating the existence of HPW and Ti on chitosan and their stability during the reaction. From the EDS (Fig. S4 $\dagger$ ), it can be seen that fresh HPW (29 wt\%)/Ti/ chitosan gave the molar ratio of W:P:Ti:C as $12: 1: 35: 100$, indicating HPW kept Keggin structure after being loaded on chitosan. After the reaction, the molar ratio of W : P : Ti : C was 12 : 1 : 34 : 102 for reused HPW/Ti/chitosan, showing little leaching of active HPW and Ti from chitosan after reaction and further demonstrating the stability of the catalyst.

In addition, the leaching test had been done just as the following: palmitic acid (0.01 mol) and HPW (29 wt\%)/Ti/ chitosan $(0.125 \mathrm{~g})$ was added into absolute methanol solution $(0.13 \mathrm{~mol})$ with stirring $1 \mathrm{~h}$ at $65^{\circ} \mathrm{C}$, then separated the catalyst and continued to react for further $6 \mathrm{~h}$. It can be found that the conversion was only increased to $35.6 \%$ from $31.4 \%$, which proved little leaching of HPW or Ti from HPW (29 wt\%)/ $\mathrm{Ti} /$ chitosan into solution during esterification reaction. This indicated that HPW/Ti/chitosan behaved as heterogeneous catalysts and little leaching of the active sites from the support. 


\section{Conclusion}

We synthesized a series of triple-functional catalyst materials HPW/Ti/chitosan containing $\mathrm{H}_{3} \mathrm{PW}_{12} \mathrm{O}_{40}$, $\mathrm{Ti}^{4+}$ and chitosan. Such HPW/Ti/chitosan hybrids exhibited Brønsted acid from $\mathrm{HPW}$, Lewis acid from $\mathrm{Ti}$ and base from $-\mathrm{NH}_{2}$ group in chitosan, which presented synergetic effect on catalyzing of esterification reaction. HPW (29 wt\%)/Ti/chitosan showed the highest conversion due to the suitable amount of HPW on chitosan. The 97.3\% conversion of palmitic acid to ester was achieved by HPW (29 wt\%)/Ti/chitosan. And little leaching of active sites of HPW and Ti from chitosan permitted it could be reused for at least six times. The loading of Ti and HPW on chitosan could provide a useful for fabrication of triple-functional and heterogeneous HPA catalysts for organic transformation.

\section{Conflicts of interest}

There are no conflicts to declare.

\section{Acknowledgements}

This work was supported by the National Natural Science Foundation of China (No. 51578119), the major projects of Jilin Provincial Science and Technology Department (20140204085GX).

\section{References}

1 M. J. Janik, B. B. Bardin, R. J. Davis and M. Neurock, J. Phys. Chem. B, 2006, 110, 4170-4178.

2 S. S. Wang and G. Y. Yang, Chem. Rev., 2015, 115, 4893-4962.

3 C. Baroi and A. K. Dalai, Ind. Eng. Chem. Res., 2014, 53, 18611-18624.

4 Q. Zhao, H. Wang, H. W. Zheng, Z. Sun, W. Shi, S. T. Wang, X. H. Wang and Z. J. Jiang, Catal. Sci. Technol., 2013, 3, 22042209.

5 M. Y. Wang, Q. W. Song, R. Ma, J. N. Xie and L. N. He, Green Chem., 2016, 18, 282-287.

6 N. R. Shiju, A. H. Alberts, S. Khalid, D. R. Brown and G. Rothenberg, Angew. Chem., 2011, 123, 9789-9793.

7 K. Liu, Y. Q. Xu, Z. X. Yao, H. N. Miras and Y. F. Song, ChemCatChem, 2016, 8, 929-937.

8 G. L. Maddikeri, A. B. Pandit and P. R. Gogate, Ind. Eng. Chem. Res., 2012, 51, 14610-14628.

9 X. M. Meng, G. Y. Chen and Y. H. Wang, Fuel Process. Technol., 2008, 89, 851-857.

10 J. Y. Yan, Y. J. Yan, S. X. Liu, J. Hu and G. L. Wang, Bioresour. Technol., 2010, 102, 4755-4758.

11 Z. Z. Wen, X. H. Yu, S. T. Tu, J. Y. Yan and E. Dahlquist, Bioresour. Technol., 2010, 101, 9570-9576.

12 I. Nurfitri, G. P. Maniam, N. Hindryawati, M. M. Yusoff and S. Ganesan, Energy Convers. Manage., 2013, 74, 395-402.

13 K. Jacobson, R. Gopinath, L. C. Meher and A. K. Dalai, Appl. Catal., B, 2008, 85, 86-91.

14 G. Baskar and R. Aiswarya, Renewable Sustainable Energy Rev., 2016, 57, 496-504.
15 N. Narkhede, S. Singh and A. Patel, Green Chem., 2015, 17, 89-107.

16 A. I. Tropecêlo, M. H. Casimiro, I. M. Fonseca, A. M. Ramos, J. Vital and J. E. Castanheiro, Appl. Catal., A, 2010, 390, 183189.

17 K. Jagadeeswaraiah, C. R. Kumar, P. S. S. Prasad and N. Lingaiah, Catal. Sci. Technol., 2014, 4, 2969-2977.

18 S. H. Zhu, X. Q. Gao, F. Dong, Y. L. Zhu, H. Y. Zheng and Y. W. Li, J. Catal., 2013, 306, 155-163.

19 R. S. Nunes, F. M. Altino, M. R. Meneghetti and S. M. P. Meneghetti, Catal. Today, 2017, 289, 121-126.

20 J. Zhao, H. Y. Guan, W. Shi, M. X. Cheng, X. H. Wang and S. W. Li, Catal. Commun., 2012, 20, 103-106.

21 V. Belessi, R. Zboril, J. Tucek, M. Mashlan, V. Tzitzios and D. Petridis, Chem. Mater., 2008, 20, 3298-3305.

22 R. C. Deltcheff, M. Fournier, R. Franck and R. Thouvenot, Inorg. Chem., 1983, 22, 207-216.

23 J. Liu, W. Y. Li, Y. G. Liu, Q. H. Zeng and S. Hong, Appl. Surf. Sci., 2014, 293, 46-54.

24 C. A. Emeis, J. Catal., 1993, 141, 347-354.

25 A. E. Kadib, K. Molvinger, C. Guimon, F. Quignard and D. Brunel, Chem. Mater., 2008, 20, 2198-2204.

26 P. K. Sahu, P. K. Sahu, S. K. Gupta and D. D. Agarwal, Ind. Eng. Chem. Res., 2014, 53, 2085-2091.

27 K. Khan and Z. N. Siddiqui, Ind. Eng. Chem. Res., 2015, 54, 6611-6618.

28 M. Yamada and A. Maeda, Polymer, 2009, 50, 6076-6082.

29 A. J. Varma, S. V. Deshpande and J. F. Kennedy, Carbohydr. Polym., 2004, 55, 77-93.

30 R. Khan and M. Dhayal, Electrochem. Commun., 2008, 10, 492-495.

31 M. Yamada and I. Honma, Electrochim. Acta, 2005, 50, 28372841.

32 S. Singh and A. Patel, J. Cleaner Prod., 2014, 72, 46-56.

33 G. Lawrie, I. Keen, B. Drew, A. C. Temple, L. Rintoul, P. Fredericks and L. Grøndahl, Biomacromolecules, 2007, 8, 2533-2541.

34 Q. Gu, J. L. Long, H. Q. Zhuang, C. Q. Zhang, Y. G. Zhou and X. X. Wang, Phys. Chem. Chem. Phys., 2014, 16, 12521-12534. 35 K. T. Karthikeyan, A. Nithya and K. Jothivenkatachalam, Int. J. Biol. Macromol., 2017, DOI: 10.1016/ j.ijbiomac.2017.03.121.

36 M. Yan, G. L. Li, C. S. Guo, W. Guo, D. D. Ding, S. H. Zhang and S. Q. Liu, Nanoscale, 2016, 8, 17828-17835.

37 R. Moucel, K. Perrigaud, J. M. Goupil, P. J. Madec, S. Marinel, E. Guibal, A. C. Gaumont and I. Dez, Adv. Synth. Catal., 2010, 352, 433-439.

38 K. Saravanan, B. Tyagi, R. S. Shukla and H. C. Bajaj, Appl. Catal., B, 2015, 172-173, 108-115.

39 R. J. Li, L. Chen and Z. C. Yan, J. Am. Oil Chem. Soc., 2012, 89, 705-711.

40 A. S. Badday, A. Z. Abdullah and K. T. Lee, Appl. Energy, 2013, 105, 380-388.

41 F. Zhang, Z. Fang and Y. T. Wang, Fuel, 2015, 150, 370-377. 42 R. A. Sheldon, M. Wallau, I. W. C. E. Arends and U. Schuchardt, Acc. Chem. Res., 1998, 31, 485-493. 